\title{
Correspondencia entre la carrera cursada y resultados del test CHASIDE en una muestra de estudiantes universitarios del primer semestre*
}

Correspondence between the chosen career and results of the CHASIDE test in a sample of university students of the first semester

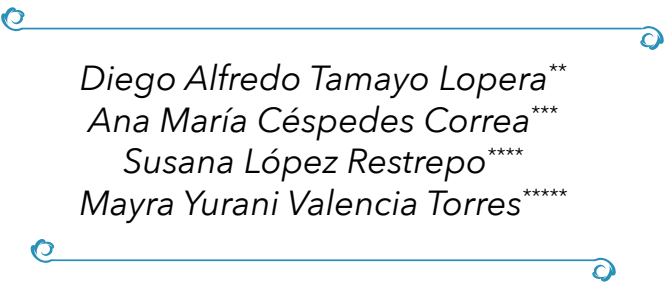

Recibido 21.11.2018• Arbitrado 09. 12. $2018 \bullet$

Aprobado 17.12. 2018

Articulo presenta resultados de investigación realizada para optar el título de psicólogo, Facultad de Ciencias Sociales, Institución Universitaria de Envigado

** Psicólogo, Magister en Neurociencias, Docente tiempo completo, Institución Universitaria de Envigado, pditalo@ gmail.com, ORCID: 0000-0001-79968871

*** Psicóloga, Institución Universitaria de Envigado, any.c.c_@hotmail.com

**** Psicóloga, Institución Universitaria de Envigado, susana_lopez92@hotmail. com

***** Psicóloga, Institución Universitaria de Envigado, yura0000@hotmail.com

\section{Resumen}

Articulo presenta resultados de estudio orientado a determinar la correspondencia entre la carrera cursada por los estudiantes de primer semestre de la Institución Universitaria de Envigado (IUE) y los resultados obtenidos por medio del Test de Orientación Vocacional CHASIDE. Se realizó un estudio descriptivo, cuantitativo y transversal. Se aplicó el Test de Orientación Vocacional CHASIDE y un cuestionario sociodemográfico a una muestra de 216 estudiantes pertenecientes a los programas tecnológicos y profesionales de una institución universitaria. Según los resultados la correspondencia con respecto al interés por el programa académico cursado fue del 58.3\% y con respecto a la aptitud fue del 44.4\%. Se encontró que de todos los programas académicos que participaron en la investigación, únicamente a la mayoría de estudiantes de Administración de Negocios Internacionales les corresponde el interés y la aptitud con su carrera. Se concluye que alrededor de la mitad de los estudiantes de primer semestre que participaron en la investigación no tienen claras las motivaciones vocacionales por las cuales eligieron el programa académico que 
cursan, misma proporción que no tiene conciencia de sus capacidades para enfrentar las demandas del programa académico elegido.

Palabras claves: aptitud vocacional, educación superior, elección profesional, interés vocacional, orientación vocacional, permanencia universitaria.

\section{Abstract}

Paper presents the results of study oriented to determine the correspondence between the career chosen by the students of the first semester of the University Institution of Envigado (IUE) and the results obtained through the CHASIDE vocational Orientation Test. A descriptive, quantitative and transversal study was carried out. The CHASIDE Vocational Orientation Test and a sociodemographic questionnaire were applied to a sample of 216 students belonging to the technological and professional programs of the IUE. According to the results, the correspondence with respect to the interest for the academic program studied was $58.3 \%$ and with respect to the aptitude was $44.4 \%$. It was found that of all the academic programs that participated in the research, only the majority of students of International Business Administration correspond the interest and aptitude with their career. It is concluded that around half of the students of the first semester of the IUE that participated in the research are not clear about the vocational motivations for which they chose the academic program that they are studying, the same proportion that is not aware of their abilities to face the demands of the chosen academic program.

Key words: higher education, professional choice, university stay, vocational aptitude, vocational interest, vocational orientation.

\section{Introducción}

Esta investigación tiene como principal propósito de estudio la elección vocacional, la cual es una de las decisiones más complejas e importantes a las que se enfrenta el individuo cuando culmina la enseñanza media y decide continuar sus estudios en la educación superior (Rodríguez y Ochoa 2012). Sin embargo, es importante señalar que la elección vocacional no es un evento que se produce únicamente en la adolescencia, en la etapa donde finaliza la educación media, pues como menciona Griffa y Moreno (2011), la elección vocacional puede darse también en la adultez joven o en la adultez media, debido a diversas circunstancias.

La elección vocacional esta guiada a partir de un proceso multicausal, ya que en esta intervienen múltiples factores determinantes tales como el con- 
texto social y familiar, género, vocación, recursos económicos, gustos e intereses. Según Ríos y Pineda (2014), Para realizar una acertada elección vocacional es fundamental implementar estrategias y programas de orientación vocacional como guía y herramienta útil, ya que la falta de ésta puede derivar en una elección inadecuada y en la ausencia de aptitud académica; además se resalta la necesidad de realizar la orientación vocacional no sólo de forma grupal sino también individual, con el fin de brindar a los estudiantes el tiempo necesario para que efectúen una adecuada elección, partiendo de sus necesidades e inquietudes individuales y a la vez se sientan acompañados y escuchados en el proceso de orientación (Higareda \& Díaz, 2014). Sin embargo y en contraposición a esto, en el artículo titulado "Orientación profesional y satisfacción vocacional" realizado por Tintaya (2016), se concluye que la orientación profesional no tiene una relación positiva o de correspondencia con la satisfacción vocacional, y que ésta aparece como un factor más casual que determinante en el desarrollo profesional de los niños y jóvenes.

La problemática con la elección vocacional surge cuando se realiza una elección vocacional desacertada, la cual puede deberse entre otras a: falta de articulación entre aptitudes e intereses (es decir que en algunas circunstancias se privilegié el interés sobre la aptitud o en otras se privilegié la aptitud sobre el interés), presiones de índole social, familiar, económicas o del contexto al momento de realizar la elección, o a factores relacionados con la orientación vocacional (poca o nula orientación vocacional, procesos de orientación vocacional dictados por personal no capacitado, tendencia de algunas instituciones de realizar los procesos de orientación vocacional de manera grupal, dejando de lado, en ocasiones, los requerimientos y necesidades propias de cada individuo).

Una de las consecuencias más desfavorables derivadas de una desacertada elección vocacional independientemente de su causa es la deserción académica, la cual se presenta como una de las principales dificultades con las que se enfrenta el Sistema de Educación Superior Colombiano, pues según estadísticas aportadas por el Ministerio de Educación Nacional de cada 100 estudiantes que ingresan a una Institución de Educación Superior alrededor de la mitad no logra finalizar su proceso académico y obtener la graduación (Ministerio de Educación Nacional [MEN], et al., 2009). Concretamente se indica que para el nivel universitario el $46.1 \%$ de los estudiantes que entran en un periodo posteriormente abandonan sus estudios y para los niveles técnico profesional y tecnológico se indica que de los alumnos que ingresan desertan más del 50\% (MEN, 2016). 
Dicho fenómeno (la deserción) puede generar entre otras, repercusiones sumamente negativas a nivel familiar, económico, académico, social, personal e institucional, es por esto que se consideró relevante investigar sobre la correspondencia entre la carrera cursada por los estudiantes de primer semestre de la Institución Universitaria de Envigado y los resultados obtenidos por medio del test de Orientación Vocacional CHASIDE, a partir del cual fue posible identificar los intereses vocacionales y las aptitudes que presentan los estudiantes frente al programa académico cursado, teniendo en cuenta que tanto intereses como aptitudes juegan un papel fundamental en dicha elección. De este modo, tanto el planteamiento como el desarrollo de la investigación podrían aportar datos que sean de utilidad para emprender acciones que conlleven al aumento de la permanencia universitaria de los estudiantes dentro de la Institución Universitaria de Envigado.

\section{Método}

El estudio tuvo un enfoque cuantitativo, nivel descriptivo y método no experimental.

\section{Participantes}

La población del estudio fue representada por 383 estudiantes de pregrado del primer semestre del nivel tecnológico y profesional de una institución universitaria. La muestra se conformó por 216 estudiantes. El rango de edad de la muestra está entre los 18 y 35 años, la media es de 22,86 años y la desviación estándar es de 6,2 años. El estrato socioeconómico de los participantes se encuentra entre 2 y 5 .

\section{Instrumentos}

Para llevar a cabo la recolección de datos se empleó el Test de Orientación Vocacional CHASIDE, desarrollado por el psicólogo Estadounidense John Holland. Este test le permite al individuo descubrir su vocación a partir de la identificación de sus intereses y aptitudes para realizar la decisión más acertada (Rojas y Rubio, 2017). El Test CHASIDE ha sido utilizado en diversos trabajos de investigación a nivel nacional (Rojas y Rubio, 2017; Orjuela y Rodríguez, 2017; Villota, Borray y Bonilla, 2018).

Se utilizó también un cuestionario sociodemográfico, el cual se sometió a evaluación por cinco expertos de la Institución Universitaria de Envigado, 
quienes realizaron sugerencias y recomendaciones que fueron tomadas en cuenta para su reformulación y posterior aplicación.

\section{Procedimiento}

Para iniciar con el trabajo de campo, se solicitó la autorización respectiva en las instancias institucionales para la aplicación de los instrumentos a la muestra fijada, dicha aplicación tuvo una duración de 40 minutos aproximadamente.

En la investigación, se tuvo en consideración la reglamentación ética estipulada en el Código Deontológico del Psicólogo (ley 1090 de 2006). Para efectos de cumplir con lo establecido en esta ley se realizó un consentimiento informado.

\section{Análisis de los datos}

Se realizó análisis univariado, para las variables cualitativas se sacaron frecuencias y porcentajes y para las variables cuantitativas medidas de tendencia central, además, se utilizaron tablas de contingencia para el análisis por grupos. En el análisis bivariado, se utilizó la prueba Chi Cuadrado de independencia para evaluar la asociación entre las variables, estableciéndose una significancia estadística con un valor $\mathrm{p}<0,05$. Se usó el programa SPSS versión 24 para los análisis.

\section{Resultados}

De los 216 estudiantes de pregrado de los programas Tecnológicos y Profesionales, $53(24.5 \%)$ pertenecen al programa de Psicología, $6(2.8 \%)$ al programa de Ingeniería Electrónica, 17 (7.9\%) al programa de Ingeniería de Sistemas, 9 (4.2\%) al programa de Tecnología de Desarrollo de Sistemas de Información, 55 (25.5\%) al programa de Derecho, 25 (11.6\%) al programa de Contaduría Pública, 24 (11.1\%) al programa de Administración de Negocios Internacionales, 21 (9.7\%) al programa de Administración Financiera y $6(2.8 \%)$ al programa de Mercadeo. Los resultados arrojan una mayor participación de los hombres 114 (52.8\%) con respecto a las mujeres 100 (46.3\%); adicionalmente de la población que hizo parte de la investigación, la mayoría pertenece al estrato socioeconómico medio 114 (67.6\%) y la minoría al estrato socioeconómico alto 8 (3.7\%) (Ver Tabla 1$)$. 
Tabla 1. Variables sociodemográficas de los estudiantes de la muestra

\begin{tabular}{cccc}
\hline $\begin{array}{c}\text { Variables } \\
\text { Sociodemográficas }\end{array}$ & Descripción & $\begin{array}{c}\text { N } \\
\text { Frecuencia }\end{array}$ & $\begin{array}{c}\text { \% } \\
\text { Porcentaje }\end{array}$ \\
\hline \multirow{2}{*}{ Sexo } & Femenino & 100 & 46.3 \\
& Masculino & 114 & 52.8 \\
\hline \multirow{2}{*}{ Estrato } & Bajo & 59 & 27.3 \\
& Medio & 146 & 67.6 \\
Programa Académico & Alto & 8 & 3.7 \\
\cline { 2 - 3 } & Psicología & 53 & 24.5 \\
& Ingeniería Electrónica & 6 & 2.8 \\
& Ingeniería de Sistemas & 17 & 7.9 \\
& Tecnología en Desarrollo de Sistemas & 9 & 4.2 \\
& de Información & 55 & 25.5 \\
& Derecho & 25 & 11.6 \\
& Contaduría Publica & 24 & 11.1 \\
& Administración de Negocios & 21 & 9.7 \\
& Internacionales & 6 & 2.8 \\
\hline
\end{tabular}

Fuente: Propia

De acuerdo a los resultados obtenidos es posible evidenciar que el nivel de Educación Superior es alto tanto en madres como en padres 38.0\% y 38.4\% respectivamente y que fueron más los alumnos que tuvieron acompañamiento por parte de sus padres o cuidadores para realizar su elección (56.0\%). Adicionalmente se puede constatar que un $52.3 \%$ de los estudiantes recibió asesoría sobre orientación vocacional; es de resaltar que la mayoría de participantes $(85.6 \%)$ considero como relevante recibir orientación vocacional para realizar una elección acertada. Se evidencia también que en un $68.1 \%$ de los participantes no se presentó influencia de los recursos económicos para realizar su elección vocacional y que un poco más de la mitad (56.5\%) de los alumnos que participaron de la investigación no repitieron ningún grado académico (Ver tabla 2). 
Tabla 2. Variables socioeducativas de los estudiantes de la muestra

\begin{tabular}{lccc}
\hline \multicolumn{1}{c}{ Variables Socioeducativas } & Descripción & N Frecuencia & \% Porcentaje \\
\hline Escolaridad del padre & Primaria & 44 & 20.4 \\
& Bachillerato & 73 & 33.8 \\
& Superior & 82 & 38.0 \\
& Ninguno & 3 & 1.4 \\
\hline Escolaridad de la Madre & Primaria & 32 & 14.8 \\
& Bachillerato & 91 & 42.1 \\
& Superior & 83 & 38.4 \\
\hline Influencia de los recursos económicos & Ninguno & 3 & 1.4 \\
\hline Asesoría sobre orientación vocacional & Si & 66 & 30.6 \\
& No & 147 & 68.1 \\
\hline Valor de la orientación vocacional & Si & 113 & 52.3 \\
& No & 98 & 45.4 \\
\hline Acompañamiento de los padres/cuidadores & Si & 185 & 85.6 \\
& Ni & 27 & 12.5 \\
\hline Repitencia de grado & No & 121 & 56.0 \\
& Si & 89 & 41.2 \\
\hline & No & 122 & 38.9 \\
& & & 56.5 \\
\hline
\end{tabular}

Fuente: Propia

Se encontró que es mayor el porcentaje de los estudiantes (69.8\%) de Psicología a los cuales les corresponde su interés con el programa académico, sin embargo, es menor el porcentaje de los que presentan correspondencia con la aptitud (52.8\%).

En Ingeniería Electrónica al $66.7 \%$ de los alumnos les corresponde su interés con su programa y al 50.0\% le corresponde la aptitud, lo que indica que la mayoría de estudiantes presentan correspondencia entre el interés y la carrera y la mitad de los alumnos presentan correspondencia en la aptitud.

En el programa de Ingeniería de Sistemas se encontró que fue mayor el porcentaje de alumnos (64.7\%) a los que no les coincidió el interés con su programa, al igual que fue mayor (52.9\%) a los que no les correspondió la aptitud.

En cuanto al programa de Tecnología en Desarrollo de Sistemas de Información, se pudo constatar que únicamente a un 33.3\% les corresponde el interés 
con su programa académico y a un $22.2 \%$ les corresponde la aptitud, lo cual indica que la mayoría de alumnos no presenta correspondencia de intereses y aptitudes con su programa.

En el programa de Derecho se puede observar que es mayor el porcentaje de alumnos (78.2\%) a los que les corresponde el interés con su carrera, así como también es mayor el porcentaje de estudiantes (70.9\%) a los que no les corresponde la carrera con la aptitud.

En cuanto a la carrera de Contaduría Pública se puede mencionar que el $64.0 \%$ de los alumnos no presento correspondencia del interés con la carrera elegida y el $52.0 \%$ si presento correspondencia en cuanto a la aptitud, lo que muestra que la mayoría de alumnos no presenta correspondencia en el interés, pero un poco más de la mitad si presenta correspondencia en la aptitud con la carrera cursada.

En la carrera de Administración de Negocios Internacionales que se da coincidencia en el interés y aptitud frente al programa, con un $54.2 \%$ y un $70.8 \%$ respectivamente.

Para el programa de Administración Financiera se encontró que fue mayor el porcentaje de estudiantes (61.9\%) que no presento correspondencia entre el interés y el programa cursado y, en cuanto a la aptitud, fue mayor el porcentaje de alumnos (52.4\%) que si tuvo correspondencia con la carrera elegida.

En la carrera de Mercadeo se halló que el 50.0\% de los participantes presento correspondencia entre el interés y la carrera elegida y que el 83.3\% no presento correspondencia entre la aptitud y dicha carrera, lo que indica que a la mitad de los alumnos les corresponde el interés con la carrera mientras que con la aptitud se encuentra muy baja correspondencia (Ver tabla 3).

Teniendo en cuenta la significación asintótica es posible evidenciar que si se presenta asociación del interés frente al programa elegido, así como también se da una asociación entre la aptitud y el programa cursado (Ver tabla 4). 
Tabla 3. Correspondencia entre el programa cursado y los intereses y aptitudes arrojados por el test de orientación vocacional CHASIDE en los estudiantes de la muestra.

\begin{tabular}{|c|c|c|c|c|}
\hline Programa & $\begin{array}{c}\text { Si } \\
\text { corresponde } \\
\text { interés } \\
\mathrm{N}(\%) \\
\end{array}$ & $\begin{array}{c}\text { No } \\
\text { corresponde } \\
\text { interés } \\
\text { N (\%) } \\
\end{array}$ & $\begin{array}{c}\mathrm{Si} \\
\text { corresponde } \\
\text { aptitud } \\
\mathrm{N}(\%) \\
\end{array}$ & $\begin{array}{c}\text { No } \\
\text { corresponde } \\
\text { aptitud } \\
\mathrm{N}(\%) \\
\end{array}$ \\
\hline Psicología & $37(69.8)$ & $16(30.2)$ & $25(47.2)$ & $28(52.8)$ \\
\hline Ingeniería Electrónica & $4(66.7)$ & $2(33.3)$ & $3(50.0)$ & $3(50.0)$ \\
\hline Ingeniería de Sistemas & $6(35.3)$ & $11(64.7)$ & $8(47.1)$ & $9(52.9)$ \\
\hline $\begin{array}{l}\text { Tecnología en Desarrollo de } \\
\text { Sistemas de Información }\end{array}$ & $3(33.3)$ & $6(66.7)$ & $2(22.2)$ & $7(77.8)$ \\
\hline Derecho & $43(78.2)$ & $12(21.8)$ & $16(29.1)$ & $39(70.9)$ \\
\hline Contaduría Publica & $9(36.0)$ & $16(64.0)$ & $13(52.0)$ & $12(48.0)$ \\
\hline $\begin{array}{l}\text { Administración de Negocios } \\
\text { Internacionales }\end{array}$ & $13(54.2)$ & $11(45.8)$ & $17(70.8)$ & $7(29.2)$ \\
\hline Administración Financiera & $8(38.1)$ & $13(61.9)$ & $11(52.4)$ & $10(47.6)$ \\
\hline Mercadeo & $3(50.0)$ & $3(50.0)$ & $1(16.7)$ & $5(83.3)$ \\
\hline Total & $126(58.3)$ & $90(41.7)$ & $96(44.4)$ & $120(55.6)$ \\
\hline
\end{tabular}

Fuente: Propia

Tabla 4. Asociación de los intereses y las aptitudes de los estudiantes de la muestra con los programas académicos elegidos.

\begin{tabular}{cccccc}
\hline Interés & Chi $\chi^{2}$ & $\begin{array}{c}\text { Significación } \\
\text { asintótica }\end{array}$ & Aptitud & Chi $\chi^{2}$ & $\begin{array}{c}\text { Significación } \\
\text { asintótica }\end{array}$ \\
\hline Programas académicos & 26.998 & $0,001^{*}$ & $\begin{array}{c}\text { Programas } \\
\text { académicos }\end{array}$ & 17,090 & $0,029^{*}$ \\
\hline
\end{tabular}

Fuente: Propia

Partiendo del análisis realizado se puede establecer que no existe asociación entre las variables sociodemográficas y educativas con el interés por el programa académico cursado por los estudiantes de la muestra. Cabe resaltar que la variable de "Orientación vocacional" no está asociada al interés por la carrera elegida por los estudiantes $(\mathrm{P}=0.823)$. Además se destaca que la variable de la "Escolaridad del padre" es la más cercana en asociación al interés por la carrera elegida $(\mathrm{P}=0.069)$ (Ver tabla 5$)$. 
Tabla 5. Asociación entre variables sociodemográficas y educativas y el interés por el programa académico de los estudiantes de la muestra

\begin{tabular}{|c|c|c|c|c|c|}
\hline Variable & & 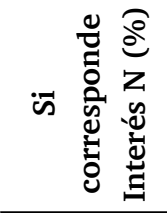 & 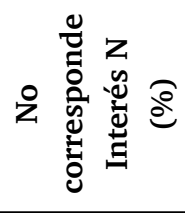 & 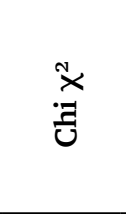 & ค \\
\hline \multirow[t]{2}{*}{ Sexo } & Femenino & $60(60.0)$ & $40(40.0)$ & & \\
\hline & Masculino & $64(56.1)$ & $50(43.9)$ & 0.326 & 0.568 \\
\hline \multirow[t]{3}{*}{ Estrato } & Bajo & $37(62.7)$ & $22(37.3)$ & & \\
\hline & Medio & $80(54.8)$ & $66(45.2)$ & & \\
\hline & Alto & $7(87.5)$ & $1(12.5)$ & 4.013 & 0.134 \\
\hline \multirow[t]{6}{*}{ Escolaridad del padre } & Primaria & $27(61.4)$ & $17(38.6)$ & & \\
\hline & Bachillerato & $45(61.6)$ & $28(38.4)$ & & \\
\hline & Técnica & $8(34.8)$ & $15(65.2)$ & & \\
\hline & Tecnología & $10(66.7)$ & $5(33.3)$ & & \\
\hline & Profesional & $24(54.5)$ & $20(45.5)$ & & \\
\hline & Ninguno & $0(0.0)$ & $3(100.0)$ & 10.215 & 0.069 \\
\hline \multirow[t]{6}{*}{ Escolaridad de la madre } & Primaria & $21(65.6)$ & $11(34.4)$ & & \\
\hline & Bachillerato & $53(58.2)$ & $38(41.8)$ & & \\
\hline & Técnica & $11(50.0)$ & $11(50.0)$ & & \\
\hline & Tecnología & $7(41.2)$ & $10(58.8)$ & & \\
\hline & Profesional & $28(63.6)$ & $16(36.4)$ & & \\
\hline & Ninguno & $2(66.7)$ & $1(33.3)$ & 3.983 & 0.552 \\
\hline \multirow[t]{2}{*}{$\begin{array}{l}\text { Influencia de los } \\
\text { recursos económicos }\end{array}$} & $\mathrm{Si}$ & $37(56.1)$ & $29(43.9)$ & & \\
\hline & No & $87(59.2)$ & $60(40.8)$ & 0.183 & 0.669 \\
\hline \multirow[t]{2}{*}{$\begin{array}{l}\text { Asesoría sobre } \\
\text { orientación vocacional }\end{array}$} & $\mathrm{Si}$ & $59(52.2)$ & $54(47.8)$ & & \\
\hline & No & $62(63.3)$ & $36(36.7)$ & 2.621 & 0.105 \\
\hline \multirow[t]{2}{*}{$\begin{array}{l}\text { Valor de la orientación } \\
\text { vocacional }\end{array}$} & $\mathrm{Si}$ & $107(57.8)$ & $78(42.2)$ & & \\
\hline & No & $15(55.6)$ & $12(44.4)$ & 0.050 & 0.823 \\
\hline \multirow[t]{2}{*}{$\begin{array}{l}\text { Acompañamiento de } \\
\text { padres/ cuidadores }\end{array}$} & $\mathrm{Si}$ & $73(60.3)$ & $48(39.7)$ & & \\
\hline & No & $47(52.8)$ & $42(47.2)$ & 1.185 & 0.276 \\
\hline \multirow[t]{2}{*}{ Repitencia de grado } & $\mathrm{Si}$ & $45(53.6)$ & 39 (46.4) & & \\
\hline & No & $73(59.8)$ & $49(40.2)$ & 0.798 & 0.372 \\
\hline \multirow{3}{*}{ edad } & Joven (18-24) & $88(55.7)$ & $70(44.3)$ & & \\
\hline & Adulto joven (25-29) & $23(67.6)$ & $11(32.4)$ & 1.837 & 0.399 \\
\hline & Adultez media (30-53) & $15(62.5)$ & $9(37.5)$ & & \\
\hline
\end{tabular}

Fuente: Propia 
Con respecto a la asociación entre variables sociodemográficas y educativas y la aptitud para el programa académico elegido, se encontró asociación de las variables "Estrato socioeconómico" $(\mathrm{P}=0,005)$; "Valor de la orientación vocacional $(\mathrm{P}=0,013)$, y "Edad" $(\mathrm{P}=0,026)$ con la "aptitud para el programa elegido" (Ver tabla 6).

Tabla 6. Asociación entre variables sociodemográficas y educativas y la aptitud para el programa académico de los estudiantes de la muestra

\begin{tabular}{|c|c|c|c|c|c|}
\hline Variable & & 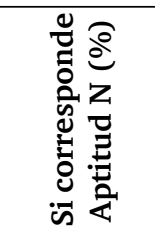 & 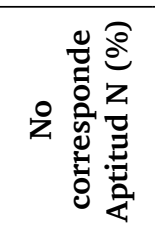 & : & 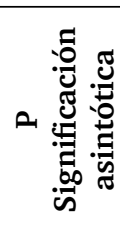 \\
\hline \multirow[t]{2}{*}{ Sexo } & Femenino & $46(46.0)$ & $54(54.0)$ & & \\
\hline & Masculino & $50(43.9)$ & $64(56.1)$ & 0,099 & 0,753 \\
\hline \multirow[t]{3}{*}{ Estrato } & Bajo & $16(27.1)$ & $43(72.9)$ & & \\
\hline & Medio & $76(52.1)$ & $70(47.9)$ & & \\
\hline & Alto & $4(50.0)$ & $4(50.0)$ & 10.635 & $0.005^{*}$ \\
\hline \multirow[t]{4}{*}{ Escolaridad del padre } & Primaria & $19(43.2)$ & $25(56.8)$ & & \\
\hline & Bachillerato & $33(45.2)$ & $40(54.8)$ & & \\
\hline & Superior & $37(45.1)$ & $45(54.9)$ & & \\
\hline & Ninguno & $2(66.7)$ & $1(33.3)$ & 0.629 & 0.890 \\
\hline \multirow[t]{4}{*}{ Escolaridad de la madre } & Primaria & $12(37.5)$ & $20(62.5)$ & & \\
\hline & Bachillerato & $42(46.2)$ & $49(53.8)$ & & \\
\hline & Superior & $38(45.8)$ & $45(54.2)$ & & \\
\hline & Ninguno & $1(33.3)$ & $2(66.7)$ & 0.942 & 0.815 \\
\hline \multirow[t]{2}{*}{$\begin{array}{l}\text { Influencia de los recursos } \\
\text { económicos }\end{array}$} & $\mathrm{Si}$ & $30(45.5)$ & $36(54.5)$ & & \\
\hline & No & $65(44.2)$ & $82(55.8)$ & 0.028 & 0.867 \\
\hline \multirow[t]{2}{*}{$\begin{array}{l}\text { Asesoría sobre orientación } \\
\text { vocacional }\end{array}$} & $\mathrm{Si}$ & $49(43.4)$ & $64(56.6)$ & & \\
\hline & No & $44(44.9)$ & $54(55.1)$ & 0.050 & 0.823 \\
\hline \multirow[t]{2}{*}{$\begin{array}{l}\text { Valor de la orientación } \\
\text { vocacional }\end{array}$} & $\mathrm{Si}$ & $88(47.6)$ & $97(52.4)$ & & \\
\hline & No & $6(22.2)$ & $21(77.8)$ & 6.133 & $0.013 *$ \\
\hline \multirow[t]{2}{*}{$\begin{array}{l}\text { Acompañamiento de } \\
\text { padres/cuidadores }\end{array}$} & $\mathrm{Si}$ & $51(42.1)$ & $70(57.9)$ & & \\
\hline & No & $42(47.2)$ & $47(52.8)$ & 0.528 & 0.467 \\
\hline \multirow[t]{2}{*}{ Repitencia de grado } & $\mathrm{Si}$ & $37(44.0)$ & $47(56.0)$ & & \\
\hline & No & $53(43.4)$ & $69(56.6)$ & 0.007 & 0.931 \\
\hline \multirow{3}{*}{ Edad } & Joven (17-24) & $66(41.8)$ & $92(58.2)$ & & \\
\hline & Adulto joven (25-29) & $22(64.7)$ & $12(35.3)$ & 7.310 & $0.026^{*}$ \\
\hline & Adultez media (30-53) & $8(33.3)$ & $16(66.7)$ & & \\
\hline
\end{tabular}

Fuente: Propia 


\section{Discusión}

Realizar una elección vocacional consiente y reflexiva es fundamental para impactar positivamente en la permanencia universitaria y en el posterior desempeño profesional del individuo. Es importante tener en cuenta que en esta elección entran en juego diversos factores tanto internos como externos, entre los cuales se puede mencionar los intereses y aptitudes, factores familiares, económicos, académicos y de orientación vocacional, los cuales pueden ser determinantes, por lo que se hace indispensable que éstos sean considerados por el estudiante para que éste asuma una posición activa en su elección (González, 1993). Dichos factores fueron tomados en cuenta durante la investigación y serán expuestos a continuación.

Los intereses y aptitudes en relación con el programa académico elegido cumplen un papel fundamental, pues en caso de que no exista interés el estudiante podría no sentirse satisfecho y motivado con su decisión, lo que podría comprometer su entrega y dedicación, o en el caso de que no se presente aptitud frente al programa, el estudiante podría verse sobrepasado por las exigencias académicas que este demande, generando gran frustración y desmotivación, poniéndose en riesgo la continuidad del proceso académico (Guzmán, 2012).

En la presente investigación fue posible identificar la correspondencia de los intereses y aptitudes que presentan los estudiantes de primer semestre de la una institución universitaria con respecto a su programa académico, a través de la aplicación del Test de Orientación Vocacional CHASIDE, el cual permitió establecer que el $58.3 \%$ de los estudiantes presenta correspondencia entre el programa académico cursado y el interés por éste, y que el 44.4\% tiene correspondencia entre la aptitud y dicho programa, evidenciando que sólo un poco más de la mitad de los alumnos presentan correspondencia entre el interés y el programa cursado y menos de la mitad presenta correspondencia entre la aptitud y su programa académico, lo que podría tener importantes implicaciones en la permanencia universitaria de los estudiantes dentro de la Institución. Esto podría ser reflejo de una de las dificultades que afronta el Sistema de Educación Superior Colombiano, ya que teniendo en cuenta las estadísticas aportadas por el Ministerio de Educación Nacional, de cada 100 estudiantes que ingresan a una institución de Educación Superior, casi la mitad no logra culminar su proceso académico y obtener la graduación, siendo estos datos similares a los enunciados anteriormente (Ministerio de Educación Nacional [MEN] et al., 2009). 
La influencia familiar por su parte como menciona Vilchis (2008) cobra gran importancia en la elección vocacional y ésta se verá favorecida siempre y cuando la familia tenga en cuenta las aptitudes e intereses de los hijos, brindando así apoyo y acompañamiento. En relación a esto, es posible establecer según los resultados de la presente investigación que a pesar de que fue mayor la cantidad de alumnos que si recibió acompañamiento por parte de sus padres o cuidadores para realizar su elección vocacional, esto no fue un factor determinante para que se presentara una asociación con el interés por el programa elegido.

Por otra parte en la investigación realizada por García et al. (2016) se afirma en cuanto a la caracterización socioeconómica, que el contexto que rodea a los estudiantes en su vida familiar y académica puede limitar o expandir su visión del futuro, lo cual puede relacionarse con la presente investigación, ya que se encontró que sí se presenta asociación del estrato socioeconómico con la aptitud por el programa cursado, teniendo en cuenta que los alumnos que presentaron mayor correspondencia son los de estrato medio, siendo el estrato bajo el que presento menor relación entre la aptitud y la carrera cursada.

Adicionalmente según Vilchis (2008), los recursos económicos pueden ser considerados como un factor determinante en la elección de una carrera, ya que en general las personas que poseen buenos recursos económicos no ven tan restringida su decisión, sin embargo, teniendo en cuenta los resultados de la presente investigación es posible establecer una diferencia significativa entre los recursos económicos y el interés que presentaron los estudiantes por su programa, es decir que no hay una asociación entre estas dos variables; no obstante a la mayoría de los estudiantes de los diferentes estratos socioeconómicos les coincidió el interés con su programa académico.

Partiendo de los resultados de esta investigación es posible resaltar que la orientación vocacional no presenta asociación con el interés por el programa académico, y que la mayoría de los estudiantes que no recibieron orientación vocacional coinciden en su interés con el programa cursado, lo cual tiene relación con lo enunciado por Tintaya (2016), quien sugiere que la orientación vocacional aparece como un factor más casual que determinante en el desarrollo vocacional de los niños y jóvenes. Lo anterior también va en línea con lo hallado en la investigación realizada por Prieto et al. (2012), donde se destaca que más de la tercera parte de los estudiantes valoraron de insuficiente y nula la orientación vocacional recibida; sin embargo, los autores de dicha investigación insisten en considerar como un factor negativo el no recibir orientación, ya que según estos la orientación vocacional es un componente importante a tener en cuenta en la formación del profesional, lo cual es afín 
con la percepción que tienen los estudiantes de la muestra del presente estudio, pues la mayoría de estos consideran la orientación vocacional como un factor relevante para realizar una adecuada elección vocacional.

Es importante resaltar que, aunque la mayoría de participantes del estudio recibió asesoría sobre Orientación Vocacional, este hecho no influyó en la correspondencia entre la aptitud y el programa elegido, lo cual podría deberse a algunas de las falencias que presenta el sistema educativo al brindar la orientación vocacional, pues como lo afirma Betancourth (2016), en ocasiones se ofrece poca orientación vocacional a los estudiantes y en otras no es impartida por personal idóneo y capacitado. En otras circunstancias, como menciona De León y Rodríguez (2008), existe falta de unificación de una metodología que permita llevar a cabo una buena orientación vocacional, o falta de credibilidad en el proceso de orientación el cual puede ser visto solo como una gestión administrativa dejando de lado la relación existente con las teorías. Por último, Higareda y Diaz (2014) mencionan que algunas veces las instituciones realizan de manera grupal los procesos de orientación vocacional, lo que puede conllevar a que se dejen de lado necesidades e inquietudes individuales, haciendo que el alumno no se perciba acompañado y asesorado en su elección.

Aunque en la presente investigación la Orientación Vocacional no fue un factor que contribuyó en la asociación del interés y la aptitud por el programa cursado, se considera relevante señalar, como menciona Cortada (1977), que la orientación vocacional contribuye en el proceso de toma de decisiones, a través de la exploración y clarificación de valores personales, es decir, aporta en la búsqueda de alternativas, en el descubrimiento de características, habilidades e intereses, y en el reconocimiento de la necesidad de una decisión.

Teniendo en cuenta la teoría evolutiva del desarrollo vocacional de Ginzberg, es posible mencionar en lo referente al "periodo realista" (periodo que va hasta los 25 años de edad, y se divide en las fases de exploración, cristalización y especificación) que es allí donde se genera la determinación de la elección vocacional, en este punto se ha superado ya el "periodo tentativo" en el que se da un reconocimiento de habilidades e intereses, los cuales son elegidos partiendo de los gustos personales y se produce además una toma de conciencia sobre la realidad del mundo, del trabajo y de la necesidad de prepararse para iniciar un camino de autonomía e independencia (Cruz, 2013). En la investigación se encontró asociación de la variable "edad" con respecto a la aptitud por el programa académico cursado, teniendo en cuenta que fueron los adultos jóvenes (personas entre los 25 y los 29 años de edad) quienes presentaron mayor aptitud por el programa que cursan, lo cual podría indicar que son estos quienes tienen más conciencia de sus habilidades para enfrentar dicho programa. Esto podría estar relacionado con lo establecido en la teoría 
de Ginzberg, la cual establece que es en esta edad donde el individuo se ha hecho consciente de lo que desea y puede y se ha identificado con una ocupación luego de valorar el éxito o fracaso al que esta le podría conllevar, lo que da lugar a la elección vocacional.

\section{Conclusiones y recomendaciones}

Según los resultados obtenidos es posible concluir que un poco más de la mitad de los estudiantes de la muestra presentan correspondencia entre el interés y el programa académico cursado y que un poco menos de la mitad de estos estudiantes presentan correspondencia entre la aptitud y el programa elegido, es decir, que alrededor de la mitad de los estudiantes que participaron en la investigación no tienen claras las motivaciones vocacionales por las cuales eligieron el programa académico que cursan, misma proporción que no tiene conciencia de sus capacidades para enfrentar las demandas del programa académico elegido, lo cual puede influir según diversos estudios en la permanencia universitaria.

Se concluye también que en la institución de la cual procede la muestra son minoría los estudiantes pertenecientes al nivel socioeconómico alto y son mayoría los pertenecientes al estrato medio, siendo estos últimos los que presentan más alta correspondencia entre la aptitud y el programa académico, sin embargo, son estos mismos los que presentan más baja correspondencia entre el interés y el programa cursado.

Es posible constatar que son las mujeres quienes tienen más correspondencia tanto en interés como en aptitud frente al programa cursado.

En lo referente a la variable edad, se encontró que fue la población joven (personas entre los 18 y 24 años) la que presentó menor interés por el programa cursado y la adulta media (personas entre los 30 y 53 años) la que presento menor aptitud por el programa elegido. Se pudo establecer también que fue a la población de adultos jóvenes (personas entre los 25 y 29 años) a la que más le coincidió el interés y la aptitud por el programa cursado.

En cuanto al acompañamiento familiar es posible establecer que éste no fue un factor determinante para que el alumno presentara mayor interés o aptitud frente a su programa académico, así como tampoco la influencia de los recursos económicos fue un factor relevante para que se presentara más interés o aptitud por el programa.

En relación a la variable escolaridad del padre y de la madre se identificó que en la mayoría de estudiantes ésta influyó para que se presentara mayor 
interés frente al programa académico cursado, teniendo en cuenta que fue en el nivel tecnológico con respecto al padre donde se presentó más correspondencia entre el interés y el programa cursado, mientras que en lo referente a la madre fue en el nivel de primaria donde se evidenció esta correspondencia. En lo concerniente a la aptitud se logró establecer que esta variable no influyó para que presentara asociación entre la aptitud y el programa académico elegido, siendo el nivel académico de primaria tanto del padre como de la madre donde se presentó menos correspondencia entre la aptitud y el programa cursado.

Recibir orientación vocacional no influyó en el interés o aptitud que presentan los alumnos por su programa académico, sin embargo, fueron más los participantes que la valoraron como relevante para realizar una adecuada elección vocacional.

Se pudo determinar que fueron los estudiantes de Derecho seguidos por los estudiantes de Psicología los que presentaron mayor correspondencia entre el interés y el programa cursado, siendo los estudiantes de Tecnología en Desarrollo de Sistemas de Información, seguidos por los estudiantes de Ingeniería de Sistemas los que presentaron más baja correspondencia entre el interés y el programa cursado.

En cuanto a la correspondencia entre la aptitud y el programa académico se determina que los estudiantes de Administración de Negocios Internacionales, seguidos de los estudiantes de Administración Financiera son los que presentan mayor correspondencia, mientras que los alumnos de Mercadeo, seguidos de los alumnos de Tecnología en Desarrollo de Sistemas de Información son los que presentan menor correspondencia.

La mayoría de los estudiantes de Ingeniería Electrónica y Tecnología en Desarrollo de Sistemas de Información no les correspondió ni el interés ni la aptitud con el programa académico, teniendo en cuenta que fueron tres los programas de la Facultad de Ingeniería los que participaron de la investigación y, como se menciona anteriormente, en dos de estos programas no se presenta tal asociación.

De todos los programas académicos que participaron en la investigación, únicamente a la mayoría de los estudiantes de Administración de Negocios Internacionales les corresponde tanto el interés como la aptitud con su carrera.

Se recomienda indagar en futuros trabajos sobre otras variables que no hayan sido tomadas en cuenta en la presente investigación y que puedan influir en la elección vocacional de los estudiantes. Al igual como profundizar en las 
diferencias o similitudes que se presentan en los factores que intervienen en la elección vocacional con respecto a la población joven y adulta.

Adicionalmente se considera pertinente determinar según los resultados arrojados por la presente investigación qué cantidad de estudiantes desertó o concluyó su proceso académico en la institución indagada, teniendo en cuenta el interés y la aptitud que presentaron los participantes en cada programa académico, esto es, determinar qué impacto se tuvo en la permanencia universitaria.

\section{Referencias}

Betancourth, L. J. (2016). Orientación Vocacional y Profesional en la Juventud Colombiana. (Monografía de posgrado). Universidad Militar Nueva Granada, Bogotá, Colombia. Recuperado de https://repository.unimilitar.edu.co/ bitstream/10654/14245/1/BetancourthS\%C3\%A1nchezLinaJhulieth.pdf

Cortada, N. (1977). El profesor y la Orientación Vocacional. México: Trilla.

Cruz, E. O. (2013). La Orientación Vocacional en el Plan de Estudios de la Institución Educativa Municipal La Arboleda, Facatativá (Tesis de maestría). Universidad Militar Nueva Granada, Bogotá, Colombia. Recuperado de http://repository.unimilitar. edu.co/bitstream/10654/11950/1/TESIS\%20EDGAR\%20ORLANDO\%20 CRUZ\%20HERNANDEZ.pdf

De León, T. Rodríguez, R. (2008). El efecto de la orientación vocacional en la elección de carrera. Revista Mexicana de Orientación Educativa. 5(13): 10-16, Recuperado de http://pepsic.bvsalud.org/scielo.php?script=sci_ arttext\&pid $=$ S1665-75272008000100004

García, G., Gáfaro, A., Bermúdez, Y., Páez, D., Prada, E., Ruiz, F.,...Rincón, G. (2016). Caracterización socioeconómica y vocacional de estudiantes de últimos grados de bachillerato en colegios públicos y privados: el caso del Área Metropolitana de Bucaramanga. Revista Lebret, (8): 265-289. doi: http://dx.doi.org/10.15332/ rl.v0i8.1698

Gonzales, V. (1993). Los niveles de integración de la motivación profesional una alternativa personologica en el estudio de la motivación. Revista Cubana de Psicología. 10(2-3), 100-103. Recuperado de http://pepsic.bvsalud.org/pdf/rcp/ v10n2-3/02.pdf

Griffa, M. C., Moreno, J. E. (2011). Claves para una psicología del desarrollo. Buenos Aires, Argentina: Lugar Editorial. 
Guzmán, D. C. (2012). Guía de orientación vocacional para estudiantes del grado undécimo, de las instituciones educativas municipales de Facatativá (Tesis de maestría). Universidad Libre, Bogotá, Colombia. Recuperado de http:// repository.unilibre.edu.co/bitstream/handle/10901/8548/1.PRELIMINARES. pdf? sequence $=1$

Higareda, J., Díaz, R. (2014). La orientación vocacional y las necesidades de los jóvenes de preparatoria. PsicoPediaHoy, 16(9): 1-10, Recuperado de http:// psicopediahoy.com/orientacion-vocacional-necesidades-jovenes-preparatoria

Medina, E. Orjuela, M. Rodríguez, J. (2017). Prototipo de una app para el diagnóstico y seguimiento vocacional de los desmovilizados como apoyo al posconflicto en Colombia. (Tesis de Postgrado). Universidad Distrital Francisco José de Caldas, Bogotá, Colombia. Recuperado de http://repository.udistrital.edu.co/ bitstream/11349/6031/1/RodriguezTovaJuanSebastian2017.pdf

Ministerio de Educación Nacional, Guzmán, C., Durán, D., Franco, J., Castaño, E., Gallón, S.,... Vásquez, J. (2009). Deserción estudiantil en la educación superior colombiana, Metodología de seguimiento, diagnóstico y elementos para su prevención. Recuperado de https://www.mineducacion.gov.co/sistemasdeinformacion/1735/ articles-254702_libro_desercion.pdf

Ministerio de Educación Nacional. (2016). Cómo va la graduación y la deserción en la educación superior. Recuperado de https://www.mineducacion.gov.co/1759/ articles-359642_recurso.pdf

Prieto, Y., Labrada, C., Hernández, C., Quesada, D. (2012). Caracterización de la orientación vocacional en estudiantes del ciclo básico de la carrera de Medicina. Educación Médica Superior, 26(2): 244-251, Recuperado de http://scielo.sld.cu/ pdf/ems/v26n2/ems07212.pdf

Ríos, R., \& Pineda, L. (2014). Factores asociados al abandono. Tipos y perfiles de abandono. Factores relacionados con deserción temprana en estudiantes de medicina. IV conferencia latinoamericana sobre el abandono en la educación superior, Medellín, Colombia. Recuperado de http://www.alfaguia.org/www-alfa/images/ PonenciasClabes/1/ponencia_152.pdf

Rodríguez, A., Ochoa, A. M. (2012). Relación entre el proceso de orientación vocacional implementado en las instituciones de educación media del departamento del Cesar y la deserción estudiantil en la Universidad Popular del Cesar (Tesis de maestría). Sistema Universitario Estatal del Caribe Colombiano, Santa Marta, Colombia. Recuperado de http://www.edunexos.edu.co/emasued/index.php/proyectos-finalizados2/ doc_download/63-relacion-entre-el-proceso-de-orientacion-vocacional 
Rojas, D. A., Rubio, L. A. (2017). Preferencias vocacionales en estudiantes de Psicología de primer año de la Corporación Universitaria Minuto de Dios VRLL (Trabajo de pregrado). Corporación Universitaria Minuto de Dios, Meta, Colombia. Recuperado de https://repository.uniminuto.edu/bitstream/handle/10656/5771/TP_ RojasDayanaAlexandra_2017.pdf? sequence $=1$ \&isAllowed $=\mathrm{y}$

Tintaya, P. (2016). Orientación profesional y satisfacción vocacional. Revista de investigación psicológica, 15: 45-58, Recuperado de http://www.scielo.org.bo/ pdf/rip/n15/n15_a04.pdf

Vilchis, M. C. (2008). Importancia de la orientación vocacional en la toma de decisiones sobre la elección de carrera en alumnos de tercer grado de secundaria (Tesis de pregrado). Universidad Pedagógica Nacional, Ciudad de México, México. Recuperado de http://200.23.113.51/pdf/25542.pdf

Villota, L. Borray, C. Bonilla, J. (2018). Características y perfiles vocacionales de los estudiantes de contaduría pública en primeros semestres. Estudio a partir de análisis de datos sociodemográficos. Adversia Revista virtual de estudiantes de contaduría pública. (21): 1-11, Recuperado de https://aprendeenlinea.udea.edu. co/revistas/index.php/adversia/article/view/337143/20792417 